\title{
ON THE ANALYTIC CONTINUATION OF MAPPING FUNCTIONS
}

\author{
BY \\ PHILIP DAVIS AND HENRY POLLAK
}

1. Introduction and summary. In this paper we study some analytic continuation properties of the mapping function of a region $B$ with an analytic boundary. We are interested in relating the continuability properties with the set of moments

$$
\mu_{m, n}=\iint_{B} x^{m} y^{n} d x d y \quad(m, n=0,1, \cdots)
$$

which is an easily obtained set of geometric quantities related to the region $B$. It is considerably more convenient to deal with the complex "moments"

$$
\sigma_{m, n}=\iint_{B} z^{m} \bar{z}^{n} d x d y ; \quad z=x+i y .
$$

In view of the relationships

$$
\begin{aligned}
& \sigma_{m, n}=\sum_{j=0, k=0}^{m, n}(i)^{m-j}(i)^{n-k} C_{m, j} C_{n, k} \mu_{j+k, m+n-j-k}, \\
& \mu_{m, n}=(-i)^{n} 2^{-m-n} \sum_{i=0, k=0}^{m, n} C_{m, j} C_{n, k} \sigma_{j+k, m+n-j-k},
\end{aligned}
$$

we are at liberty to pass from one set of moments to another.

The relevance of the moments (1) to plane sets $S$ is that they form a "complete set" of domain functionals when the domains $S$ have been suitably restricted. We may define this notion as follows: Let $\subseteq$ designate a family of sets $S$ and let $F_{n}=F_{n}(S) \quad(n=1,2, \cdots)$ be a sequence of set functionals each of which is defined for all $S \in \subseteq$. The sequence $\left\{F_{n}\right\}$ will be called complete for $\mathbb{S}$ if

$$
F_{n}(S)=F_{n}(T), \quad(n=1,2,3, \cdots), S, T, \in, \subseteq
$$

implies $S=T$. The equation $S=T$ may hold a.e., or it may hold in the stronger sense that each point of $S$ is a point of $T$ and vice versa.

A relationship between complete sets of domain functionals and complete sets of functions is easily found.

THEOREM. Let $\sqrt{5}$ designate the family of plane sets which are measurable and which lie in a fixed circle $C$. Let $L(C)$ designate the class of functions which

Presented to the Society September 1, 195.5; received by the editors September 15, 1956. 
are defined over $C$ and are integrable. Let $f_{n}(x, y) \in L(C)$. A necessary and suffcient condition that

$$
\iint_{S} f_{n}(x, y) d x d y=\iint_{T} f_{n}(x, y) d x d y, \quad(n=1,2,3, \cdots)
$$

imply $S=T$ a.e., is that the sequence $\left\{f_{n}\right\}$ be complete in $L(C)$.

Proof. Sufficiency. Designate the characteristic function of a set $S$ by $C_{S}(x, y)$. Assuming the above equality,

$$
\begin{aligned}
\iint_{C} C_{S}(x, y) f_{n}(x, y) d x d y & =\iint_{S} f_{n}(x, y) d x d y=\iint_{T} f_{n}(x, y) d x d y \\
& =\iint_{C} C_{T}(x, y) f_{n}(x, y) d x d y .
\end{aligned}
$$

Thus,

$$
\iint_{C}\left(C_{S}-C_{T}\right) f_{n} d x d y=0 \quad(n=1,2,3, \cdots) .
$$

Since $\left\{f_{n}\right\}$ is complete in $L(C)$, and $C_{S}-C_{T}$ is in $L(C)$, this implies that $C_{S}-C_{T}=0$ a.e. Hence, $S=T$ a.e.

Necessity. Let $S \in \subseteq$ and let $\theta$ be a set of measure 0 . Then,

$$
\iint_{S} f_{n} d x d y=0 \quad(n=1,2,3, \cdots),
$$

implies

$$
\iint_{S} f_{n} d x d y=\iint_{\theta} f_{n} d x d y \quad(n=1,2,3, \cdots) .
$$

But this implies that $S=\theta$ a.e. Hence, $C_{S}=0$ a.e. Therefore, $\left\{f_{n}\right\}$ is complete for the set of characteristic functions. Since linear combinations of characteristic functions are dense in $L(C)$, it follows that $\left\{f_{n}\right\}$ is complete for $L(C)$.

We may restrict membership in $\subseteq$ further and eliminate the necessity for writing "almost everywhere" by producing a family for which $S, T, \in, \subseteq, S$ $=T$ a.e., implies $S=T$. For instance, the following family has this property. Let $\subseteq$ designate the family of open sets $S$ lying in a fixed circle $C$ and such that there are exterior points of $S$ in any neighborhood of any boundary point of $S$.

By way of proof, suppose that $S=T$, a.e. We wish to show $S=T$. Suppose there were a point $P$ in $S$ but which is not in $T$. Such a point cannot be an exterior point of $T$. For then, we could find a neighborhood $N$ of $P$ which is contained in $S$ and in the exterior of $T$. Thus $S$ and $T$ would differ by more than a set of measure 0 . Such a point $P$ cannot also be a boundary point of 
$T$. For in such a case we could, by hypothesis, find a neighborhood $N$ of $P$ which is interior to $S$ and which contains a point $P^{\prime}$ which is in the exterior of $T$. Then we could find a neighborhood $N^{\prime}$ of $P^{\prime}$ which is interior to $S$ and exterior to $T$. This again is impossible since $S$ and $T$ would differ by more than a set of measure 0. The assumption that $P$ is not in $T$ is therefore false. Similarly, if $P$ is in $T$, we may prove it is in $S$. Therefore $S=T$.

CoROLlaRy. Let $S$ and $T$ be bounded open sets which possess exterior points in any neighborhood of any boundary point. Then

$$
\iint_{S} x^{m} y^{n} d x d y=\iint_{T} x^{m} y^{n} d x d y \quad(m, n=0,1, \cdots)
$$

implies $S=T$.

Proof. This is now a consequence of the completeness of the powers in $L(C)$.

Before we proceed with the general exposition, we shall give a short summary of the methods and results. For this purpose, we shall think of a simplyconnected domain $B$ with an analytic boundary; some of the results in the body of the paper, however, are for multiply connected regions.

To begin with the methods, let us see briefly why the moments of the domain $B$ and the continuability of various mapping functions ought to be related to one another. We start by considering the transform

$$
f(z)=T g(z) \equiv \frac{1}{\pi} \iint_{B} \frac{[g(w)]-d A_{w}}{z-w},
$$

where $z$ is outside the closure of $B$. On the one hand, many properties of $f(z)$ are contained in the coefficients of the expansion

$$
f(z)=\frac{1}{\pi} \sum_{j=0}^{\infty} \frac{1}{z^{j+1}} \iint_{B} w^{j}[g(w)]^{-} d A_{w} .
$$

In the special case in which $g(w)=w^{n}$, the coefficients are just moments of $B$.

On the other hand, Green's theorem gives

$$
f(z)=\frac{1}{2 \pi i} \int_{b} \frac{\lfloor h(w)]-d w}{z-w} ;
$$

where $h=\int g d w$. If we define $\bar{h}(w)=[h(\bar{w})]-$ for $w \in \bar{b}$, then

$$
f(z)=\frac{1}{2 \pi i} \int_{b} \frac{\bar{h}(\bar{w}) d w}{z-w}=\frac{1}{2 \pi i} \int_{b} \frac{\bar{h}(S(w)) d w}{z-w},
$$

where $S(w)$ is what we have called the Schwarz function of $b$, i.e., the function on $b$ such that $w=S(w)$. If $b$ is analytic, then $S(w)$ is, of course, analytic in a neighborhood of $b$, and 


$$
S(w)=\bar{m}\left\{\frac{1}{M(w)}\right\}
$$

where $M$ is any function mapping $b$ onto the circumference of the unit circle, with $m$ as its inverse. If we pick for $M$ a particular mapping function of $B$, this procedure gives, then, relationships between the continuability of $m$, through the continuability of $S$, to the moments of $B$.

Another important tool is the inversion formula for the transform $T$, given by

$$
[g(w)]^{-}=\frac{1}{2 i} \int_{b} K_{B}(z, \bar{w}) f(z) d z,
$$

where $K_{B}$ is the Bergman kernel of $B$.

Theorem 4 states that if $m(z)$ maps the unit circle onto $B$ such that $m(0)$ $=0$, then a necessary and sufficient condition for $\vec{h}(\vec{m}(z))$ to be an entire function is that

$$
\lim _{n \rightarrow \infty}\left|\iint_{B} z^{n}[g(z)]-d A\right|^{1 / n}=0 .
$$

Here, as defined previously, $h$ is an indefinite integral of $g$. If we choose $g(z)$ $=z^{k}$, we obtain that a necessary and sufficient condition for $(\bar{m}(z))^{k+1}$, and hence $(m(z))^{k+1}$, to be entire is that

$$
\lim _{n \rightarrow \infty}\left|\iint_{B} z^{n} \bar{z}^{k} d A\right|^{1 / n}=0 .
$$

Other choices of $g$ give further theorems about $m$. Later theorems discuss the possibilities for $h(m(z))$ to be polynomial, and to be an entire function of a specified order $\alpha$.

Finally, we study the meaning of

$$
\limsup _{n \rightarrow \infty}\left|\iint_{B} z^{n}[g(z)]^{-} d A\right|^{1 / n}
$$

itself. For regions with an analytic boundary, this may be identified as the parameter on the furthest curve of a family to which a certain function continues; for domains with a nonanalytic boundary, this limit has not been identified thus far.

2. Preliminary identities and expansions. We shall assume that we are dealing with a finite region $B$ (see Fig. 1) lying in the complex $z=x+i y$ plane and bounded by $N+1$ analytic Jordan curves $b_{0}, b_{1}, \cdots, b_{N}$. It is assumed that $b_{0}$ surrounds the others. The finite simply connected region which each $b_{k}, k \geqq 1$, bounds is designated by $B_{k}$. $B_{0}$ will designate the infinite region whose boundary is $b_{0}$. The following notation will be employed: for a point 


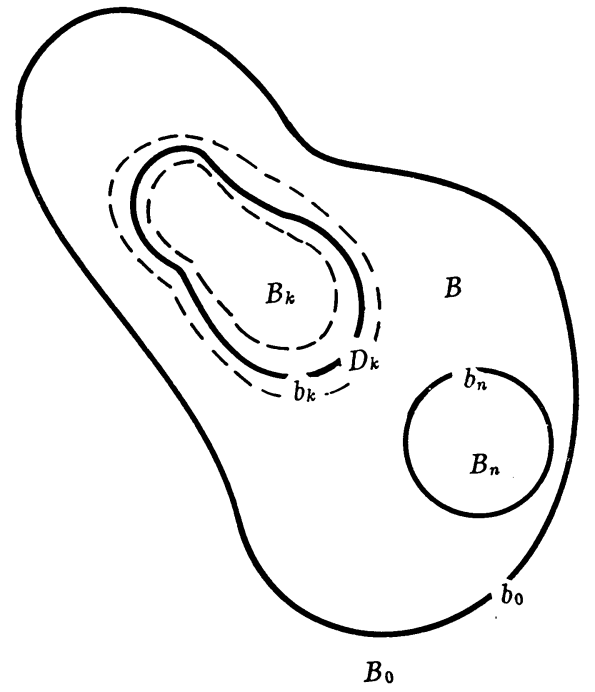

FIG. 1

set $S, \hat{S}$ will designate its closure, $\widetilde{S}$ will designate its complement with respect to the Riemann sphere, and $\bar{S}$ will designate the conjugate of $S$. That is, $\bar{S}$ is the reflection of $S$ in the line $y=0$. Our grasp on the moments (2) (or (1)) is through the transform

$$
f(z)=T(g(w))=\frac{1}{\pi} \iint_{B} \frac{[g(w)]^{-}}{z-w} d A_{w}
$$

Here $d A_{w}$ is the area element in the $w$ variable. Certain aspects of the related transform

$$
f(z)=\frac{1}{\pi} \iint_{B} \frac{[g(w)]^{-}}{(z-w)^{2}} d A_{w}
$$

have been studied by S. Bergman and M. Schiffer [1], I.E. Block [2] and A. Beurling [unpublished]. Our interest in this transform lies in an altogether different direction.

We shall restrict the functions $g$ to which $T$ is applied to those with the following properties: (a) they are regular in $B$ and continuous in $\hat{B}$, (b) they possess an integral

$$
h(z)=\int_{z_{0}}^{z} g(t) d t
$$

which is a single-valued analytic function throughout $B$.

By the complex form of Green's theorem, (4) may be rewritten in the form 


$$
f(z)=\frac{1}{2 \pi i} \int_{b} \frac{[h(w)]^{-}}{z-w} d w=\frac{1}{2 \pi i} \sum_{k=0}^{N} \int_{b_{k}} \frac{[h(w)]^{-} d w}{z-w} .
$$

It is clear from (4) or from (7) that, starting from a given $g(w)$, the transform $T$ defines $N+1$ analytic functions in $B_{0}, B_{1}, \cdots, B_{N}$. It is clear, moreover, from (7), that it also defines an analytic function in $B$ itself. These $N+2$ analytic functions are not continuations of one another. Nevertheless, the set of functions defined in this way will be designated simply by $f(z)$. To avert any possible ambiguity, we shall also use $f_{0}(z), \cdots, f_{N}(z)$, to designate the "components" of $f(z)$.

Our first object is to obtain some representations for $T(g)$. Let $D_{k}$ $(k=0,1, \cdots, N)$ be a region which contains $b_{k}$. In particular, $D_{k}$ may be an annulus-like strip which contains $b_{k}$. Let the function

$$
\zeta=M_{k}(w) \text { with inverse } w=m_{k}(\zeta)
$$

be regular and schlicht in $D_{k}$ and map $b_{k}$ onto $|\zeta|=1$. Then from (7) we have

$$
f(z)=\frac{1}{2 \pi i} \sum_{k=0}^{N} \int_{|\zeta|=1} \frac{h\left(m_{k}(\zeta)\right)}{z-m_{k}(\zeta)} m_{k}^{\prime}(\zeta) d \zeta .
$$

We now employ the following notation: let $t(z)$ be regular in a region $S$. By $\bar{t}(z)$ we shall mean the "reflection" of $t(z)$, that is, $\bar{t}(z)$ is that function regular in $\bar{S}$ and defined by

$$
\bar{t}(z)=[t(\bar{z})]-.
$$

Since $\zeta \bar{\zeta}=1$ on $|\zeta|=1$, we have

$$
\left[m_{k}(\zeta)\right]^{-}=\left[\bar{m}_{k}\right]^{-}\left(\frac{1}{\zeta}\right) \text { on }|\zeta|=1 .
$$

Hence, (9) can be written in the form

$$
f(z)=\frac{1}{2 \pi i} \sum_{k=0}^{N} \int_{|\zeta|=1} \frac{\bar{h}\left(\bar{m}_{k}(1 / \zeta)\right)}{z-m_{k}(\zeta)} m_{k}^{\prime}(\zeta) d \zeta .
$$

The paths of integration in (12) may now be deformed provided that no singularities of the integrand are encountered. If the inverse transformations $\zeta=M_{k}(w)$ are applied to (12), there results

$$
f(z)=\frac{1}{2 \pi i} \sum_{k=0}^{N} \int_{b_{k}} \frac{\bar{h}\left(\bar{m}_{k}-\left(1 / M_{k}(w)\right)\right)}{z-w} d w .
$$

3. The Schwarz function for an analytic curve. The representation (13) may be simplified. Let us examine the function which appears in the integrand of (13): $S(w)=\bar{m}(1 / M(w))$. (We have deleted the indexing). This function is, first of all, analytic in an annulus-like region which contains $b$ in its inte- 
rior. It ostensibly depends upon the particular pair of mapping functions $(M, m)$ which have been selected. But we can show that, in reality, it is independent of these and depends solely upon the analytic Jordan contour $b$. To show this, observe that for $z \in b$, we have $|M(z)|=1$, hence by (11),

$$
\bar{m}(1 / M(z))=[m(M(z))]^{-}=\bar{z} ; \quad z \in b .
$$

That is, $S(z)$ is an analytic function of $z$ which at the points $z \in b$ takes on the values $\bar{z}$. But, an analytic function is uniquely determined by its values along a curve. Thus, $\bar{m}(1 / M(z))$ depends only upon $b$.

An analytic curve may be characterized by a mapping function onto the unit circle. However, such a function is not unique, while the function $S(z)$ for which

$$
S(z)=\bar{z}
$$$$
z \in b
$$

is unique. We call this function the Schwarz function for the analytic curve $b$.

$$
S(z)=\bar{m}(1 / M(z)) .
$$

Thus, for the unit circle,

$$
S(z)=1 / z .
$$

However, it can also be obtained explicitly in many cases where a mapping function is not immediately available. Thus, e.g., consider the algebraic curve $C$ :

$$
x^{4}+y^{4}=1 .
$$

Writing $x=(z+\bar{z}) / 2, y=(z-\bar{z}) / 2 i$, we have on $C$,

$$
(z+\bar{z})^{4}+(z-\bar{z})^{4}=2 z^{4}+12 z^{2} \bar{z}^{2}+2 \bar{z}^{4}=16 ;
$$

whence, solving for $\bar{z}$,

$$
S(z)=\left(-3 z^{2}+2^{3 / 2}\left(z^{4}+1\right)^{1 / 2}\right)^{1 / 2} .
$$

In $\$ 10$, we indicate some additional properties of the function $S(z)$.

4. Further representations for $T(g)$. For each contour $b_{k}(k=0,1, \cdots, N)$ let us introduce its Schwarz function $S_{k}(z)$. Then, (13) becomes

$$
f(z)=\frac{1}{2 \pi i} \sum_{k=0}^{N} \int_{b_{k}} \frac{\bar{h}\left(S_{k}(w)\right)}{z-w} d w .
$$

This representation can be obtained directly from (7) and (15).

Let $a$ designate a point of the finite plane which does not lie on $b$. Let $d_{a}=\operatorname{dist}(a, b)$. Then, for $|z-a|<d_{a}$ we have

$$
(z-w)^{-1}=(a-w)^{-1} \sum_{j=0}^{\infty}((z-a) /(w-a))^{j}
$$


uniformly for $w \in b$ and so

$$
f(z)=-\frac{1}{2 \pi i} \sum_{j=0}^{\infty}(z-a)^{j} \sum_{k=0}^{N} \int_{b_{k}} \frac{\bar{h}\left(S_{k}(w)\right)}{(w-a)^{j+1}} d w .
$$

By expanding $(z-w)^{-1}$ in negative powers of $z$, we obtain a representation of $f(z)$ valid in a neighborhood of $z=\infty$;

$$
f_{0}(z)=\frac{1}{2 \pi i} \sum_{j=0}^{\infty} z^{-j-1} \sum_{k=0}^{N} \int_{b_{k}} w^{j} \bar{h}\left(S_{k}(w)\right) d w .
$$

If the point $a,|a|<\infty$, is located in $B_{0}, B_{1}, \cdots$, or $B_{N}$, then a similar process applied to (4) yields

$$
f(z)=-\sum_{j=0}^{\infty}(z-a)^{i} \frac{1}{\pi} \iint_{B} \frac{[g(w)]-d A_{w}}{(w-a)^{j+1}},
$$

and when $a=\infty$,

$$
f_{0}(z)=\sum_{j=0}^{\infty} z^{-j-1} \frac{1}{\pi} \iint_{B} w^{j}[g(w)]-d A .
$$

By comparing (22) and (24), (23) and (25), there are obtained the identities

$$
\begin{aligned}
& \frac{1}{\pi} \iint_{B} \frac{[g(w)]^{-} d A_{w}}{(w-a)^{j+1}}=\frac{1}{2 \pi i} \sum_{k=0}^{N} \int_{b_{k}} \frac{\bar{h}\left(S_{k}(w)\right)}{(w-a)^{j+1}} d w \\
& \frac{1}{\pi} \iint_{B} w^{j}[g(w)]^{-} d A=\frac{1}{2 \pi i} \sum_{k=0}^{N} \int_{b_{k}} w^{i} \bar{h}\left(S_{k}(w)\right) d w .
\end{aligned}
$$

These identities can also be established by applying Green's theorem directly to the left hand members and using (16). By passing to the plane of the unit circle, and using (8) and (16), we have

$$
\begin{aligned}
& \frac{1}{\pi} \iint_{B} \frac{[g(w)]-d A_{w}}{(w-a)^{j+1}}=\frac{1}{2 \pi i} \int_{|\zeta|=1} \sum_{k=0}^{N} \frac{\bar{h}\left(\bar{m}_{k}(1 / \zeta)\right)}{\left(m_{k}(\zeta)-a\right)^{j+1}} m_{k}^{\prime}(\zeta) d \zeta_{k} \\
& \frac{1}{\pi} \iint_{B} w^{j}[g(w)]-d A=\frac{1}{2 \pi i} \int_{|\zeta|=1} \sum_{k=0}^{N}\left[m_{k}(\zeta)\right]^{j} \bar{h}\left(\bar{m}_{k}\left(\frac{1}{\zeta}\right)\right) m_{k}^{\prime}(\zeta) d \zeta .
\end{aligned}
$$

As a special case of $\left(27^{\prime}\right)$, we select $g(w)=w^{n}$. This yields

$$
\text { (28) } \frac{1}{\pi} \iint_{B} w^{i_{\bar{w}} \bar{w}^{n}} d A=\frac{1}{2 \pi i(n+1)} \int_{|\zeta|=1} \sum_{k=0}^{N}\left[m_{k}(\zeta)\right]^{j}\left[\bar{m}_{k}(1 / \zeta)\right]^{n+1} m_{k}^{\prime}(\zeta) d \zeta \text {. }
$$

5. Representation of $T$ in terms of polynomials of Faber type. Assume that the origin lies in $B$ and consider the set of functions

$$
\left[\Phi_{n}(z)\right]^{-}=\left.\frac{\partial^{n}}{\partial w^{n}} K_{B}(\bar{z}, w)\right|_{w=0} \quad(n=0,1, \cdots)
$$


where $K_{B}(z, \bar{w})$ is the Bergman kernel function( $\left.{ }^{1}\right)$ for the region $B$. If the functions $\Phi_{n}$ are orthonormalized with respect to the inner product $(f, g)$ $=\iint_{B} f \bar{g} d A$, then there is obtained a set of functions $\Phi_{n}^{*}(z)$ which are complete and orthonormal for the class $L^{2}(B)$. For simply connected regions, the functions $\Phi_{n}^{*}$ reduce to

$$
\Phi_{n}^{*}(z)=\left(\frac{n+1}{\pi}\right)^{1 / 2} M^{\prime}(z)[M(z)]^{n}
$$

where $M(z)$ is the interior mapping function of $B\left({ }^{2}\right)$. For a fixed $z$ in the exterior of $B, 1 /(z-w)$ is a regular function of $w, w \in \hat{B}$ and hence, we have

$$
\frac{1}{z-w}=\sum_{n=0}^{\infty} \alpha_{n} \Phi_{n}^{*}(w) ; \quad \alpha_{n}=\alpha_{n}(z)=\iint_{B} \frac{\left[\Phi_{n}^{*}(w)\right]^{-}}{z-w} d A_{w} .
$$

The convergence of (30) is uniform and absolute in $B$. The coefficients $\alpha_{n}(z)$ may, in view of the 2 nd equation of (30) and (29), be written in the form

$$
\alpha_{n}(z)=\frac{1}{z} P_{n}\left(\frac{1}{z}\right)
$$

where $P_{n}(z)$ is a polynomial $\left({ }^{3}\right)$ of degree $n$. Thus, we may write

$$
\frac{1}{z-w}=\sum_{n=0}^{\infty} \frac{1}{z} P_{n}\left(\frac{1}{z}\right) \Phi_{n}^{*}(w) .
$$

In the equation (32), it is not necessary for $z$ to lie exterior to $B$. Take any $z \neq 0$, then there is a region $C_{r}: G(z, 0)>-\log r>0,0<r<1$ containing the origin such that $z$ is exterior to $C_{r} . G(z, 0)$ designates the Green's function for the region $B$ with pole at $z=0$. For such a $z,(z-w)^{-1}$ as a function of $w$ is regular in $C_{r}$. Hence the series (32) converges $\left({ }^{4}\right)$ uniformly and absolutely for $w$ in $C_{r}$. We may conclude further, that if $S$ is any point set which is bounded away from $z=0$, then (32) converges absolutely and uniformly for all $z$ in $S$ and for all $w$ in a sufficiently small neighborhood $N$ of the origin. Take $S$ as $b$. Then for any function $t(z)$ which is regular in $B$ and continuous in $\hat{B}$ we have, for $w \in N$,

$$
t(w)=\frac{1}{2 \pi i} \int_{b} \frac{t(z)}{z-w} d z=\sum_{n=0}^{\infty}\left(\frac{1}{2 \pi i} \int_{b} \frac{1}{z} P_{n}\left(\frac{1}{z}\right) t(z) d z\right) \cdot \Phi_{n}^{*}(w) .
$$

But, in view of the orthogonality of the set $\Phi^{*}$, we must have, in addition,

(1) S. Bergman, [3, p. 21].

(2) Bergman [3, p. 10]. See also Walsh and Davis [7] where the functions have been generalized and their convergence properties extended.

$\left(^{3}\right)$ These polynomials are of Faber type. See Davis and Pollak [4, p. 661].

(4) Walsh and Davis [7, p. 9]. 


$$
t(w)=\sum_{n=0}^{\infty}\left(\iint_{B} t(w) \Phi_{n}^{*}(w) d A\right) \Phi_{n}^{*}(w) .
$$

By comparing (33) and (34), we obtain

$$
\iint_{B} t(w)\left[\Phi_{n}^{*}(w)\right]-d A=\frac{1}{2 \pi i} \int_{b} \frac{1}{z} P_{n}\left(\frac{1}{z}\right) t(z) d z .
$$

Take now, $t(w)=\Phi_{m}^{*}(w)$. These functions are, in view of (29) and the stated hypotheses on $b$, regular on $b$. Hence we have

$$
\iint_{B} \Phi_{m}^{*}(w)\left[\Phi_{n}^{*}(w)\right]-d A=\delta_{m n}=\frac{1}{2 \pi i} \int_{b} \frac{1}{z} P_{n}\left(\frac{1}{z}\right) \Phi_{n}^{*}(z) d z .
$$

From (32), the transform (4) can be written as

$$
f(z)=\frac{1}{\pi} \sum_{n=0}^{\infty}\left(\iint_{B}[g(w)]-\Phi_{n}^{*}(w) d A\right) \frac{1}{z} P_{n}\left(\frac{1}{z}\right) z \in B_{0}, B_{1}, \cdots, B_{N} .
$$

6. An inversion formula for $T$. We now derive an inversion formula for $T(g)$ under the assumption that $g$ is analytic in $\hat{B}$. Under this assumption, $h$ is also regular in $\hat{B}$, and hence, we may find contours $b_{k}^{*}(k=0,1, \cdots, N)$, $b_{k}^{*}$ lying in $B, b_{1}^{*}, \cdots, b_{N}^{*}$ surrounding $b_{1}, \cdots, b_{N}$ respectively, and $b_{0}^{*}$ surrounded by $b_{0}$, and such that

$$
f(z)=\frac{1}{2 \pi i} \sum_{k=0}^{N} \int_{b_{k}^{*}} \frac{\bar{h}\left(S_{k}(w)\right)}{z-w} d w .
$$

(Cf. (21)). Hence, $f(z)$ may be continued analytically across $b_{k}$. (That is, each of the $N+1$ analytic components of $f, f_{k}$, can be continued across its appropriate $\left.b_{k},(k=0,1, \cdots, N)\right)$. Consider now,

$$
\Phi_{m}^{*}(z) f(z)=\frac{\Phi_{n}^{*}(z)}{2 \pi i} \sum_{k=0}^{N} \int_{b_{\boldsymbol{k}}^{*}} \frac{\bar{h}\left(S_{k}(w)\right)}{z-w} d w .
$$

Integrating (39) over $b$ we obtain

$$
\begin{gathered}
\int_{b} \Phi_{m}^{*}(z) f(z) d z=\frac{1}{2 \pi i} \int_{b} \Phi_{m}^{*}(z) \sum_{j=0}^{N} \int_{b_{j}^{*}} \frac{\bar{h}\left(S_{j}(w)\right)}{z-w} d w d z \\
=\sum_{j=0}^{N} \int_{b_{j}^{*}} \bar{h}\left(S_{j}(w)\right) d w \frac{1}{2 \pi i} \int_{b} \frac{\Phi_{m}^{*}(z)}{z-w} d z=\sum_{j=0}^{N} \int_{b,} \bar{h}\left(S_{j}(w)\right) \Phi_{m}^{*}(w) d w \\
=\sum_{j=0}^{N} \int_{b_{j}} \bar{h}\left(S_{j}(w)\right) \Phi_{m}^{*}(w) d w=2 i \iint_{B}[g(w)]^{-} \Phi_{m}^{*}(w) d A .
\end{gathered}
$$

The last equality follows by Green's theorem. Now, 


$$
g(w)=\sum_{n=0}^{\infty}\left(\iint_{B} g(w)\left[\Phi_{n}^{*}(w)\right]-d A\right) \Phi_{n}^{*}(w), \quad w \in B
$$

so that by $(40)$,

$$
[g(w)]^{-}=\frac{1}{2 i} \sum_{n=0}^{\infty}\left(\int_{b} \Phi_{n}^{*}(z) f(z) d z\right)\left[\Phi_{n}^{*}(w)\right]^{-}, \quad w \in B .
$$

Now, $K_{B}(z, w)=\sum_{n=0}^{\infty} \Phi_{n}^{*}(z)\left[\Phi_{n}^{*}(w)\right]^{-}$, this series converging uniformly for $z \in B$ and $w$ confined to any closed region contained in $B$. Thus,

$$
[g(w)]^{-}=\frac{1}{2 i} \int_{b} K_{B}(z, \bar{w}) f(z) d z, \quad w \in B .
$$

We are now in a position to establish the following result $\left({ }^{5}\right)$.

THEOREM 1. The function $g(z)$ can be continued analytically across all components of $b$ if and only if each component function

$$
f_{k}(z)=\frac{1}{\pi} \iint_{B} \frac{[g(w)]^{-}}{z-w} d A_{w}, \quad z \in B_{k}
$$

can be continued analytically across $b_{k}$ to the interior of $B$. In such a case, the inverse of $T$ is given by

$$
[g(w)]^{-}=\frac{1}{2 i} \int_{b} K_{B}(z, \bar{w}) f(z) d z
$$

where the values of $f_{k}$ are used on $b_{k}$ in forming (45).

Proof. We have already shown that if $g(z)$ is regular in $\hat{B}$, then $f_{k}(z)$ continues analytically across $b_{k}$ and (45) holds. Suppose, conversely, that $f_{k}(z)$ comes from a $g(w)$ through $(44)$ and that each $f_{k}$ continues analytically across $b_{k}$. Define a function $g^{*}(w)$ by means of (45). That is,

$$
\left[g^{*}(w)\right]^{-}=\frac{1}{2 i} \int_{b} K_{B}(z, \bar{w}) f(z) d z .
$$

By our regularity hypothesis, we may find contours $b_{k}^{*}$ lying interior to $B$ on which $f_{k}(z)$ is still regular. Thus,

$$
\left[g^{*}(w)\right]^{-}=\frac{1}{2 i} \int_{b^{*}} K_{B}(z, \bar{w}) f(z) d z .
$$

Now, for $z$ on $b^{*}$, it is known that $K_{B}(z, \bar{w})$ is an analytic function of $w$ which

(5) These matters have been discussed in Davis and Walsh [5] for simply connected domains and from a different point of view. 
may be continued analytically across $b_{k}(k=0,1, \cdots, N)$. Thus, by (47), $g^{*}(w)$ must also have this property. For $t$ in $\widetilde{B}$,

$$
T\left(g^{*}\right)=\frac{1}{\pi} \iint_{B} \frac{\left[g^{*}(w)\right]^{-}}{z-w} d A_{w}=\frac{1}{2 \pi i} \iint_{B} \frac{d A_{w}}{t-w} \int_{b^{*}} K_{B}(z, \bar{w}) f(z) d z .
$$

Since both integrands are regular over the indicated sets, we may invert the order of integration and obtain

$$
\begin{aligned}
T\left(g^{*}\right) & =\frac{1}{2 \pi i} \int_{b^{*}} f(z) d z \iint_{B} \frac{1}{t-w} K_{B}(z, \bar{w}) d A_{w} \\
& =\frac{1}{2 \pi i} \int_{b^{*}} \frac{f(z) d z}{t-z}=\frac{1}{2 \pi i} \int_{b} \frac{f(z) d z}{t-z} \\
& =\sum_{k=0}^{N} \frac{1}{2 \pi i} \int_{b_{k}} \frac{f_{k}(z)}{t-z} d z .
\end{aligned}
$$

Now, let $t \in B_{j}$. Then, $(2 \pi i)^{-1} \int_{b_{j}}\left(f_{j}(z) /(t-z)\right) d z=f_{j}(t)$ while

$$
(2 \pi i)^{-1} \int_{b_{k}}\left(f_{k}(z) /(t-z)\right) d z=0 \text { for } k \neq j .
$$

Thus we have, $T\left(g^{*}\right)=f(z)$, component by component. Thus, $T\left(g-g^{*}\right)=0$. It follows from (37) that $\iint_{B} \Phi_{n}^{*}\left[g-g^{*}\right]-d A=0(n=0,1, \cdots)$. Since $\left\{\Phi_{n}^{*}\right\}$ is complete in $L^{2}(B)$, this implies that $g \equiv g^{*}$.

7. General continuation theorems. The transform $f=T(g)$ is known initially to define $N+1$ analytic functions in the $N+1$ components of $\widetilde{B}$. If it is assumed that $g$ is regular in $\hat{B}$, then it follows from the last section that each component of $f$ may be continued analytically across the corresponding $b_{k}$. In the present section we study the singularity structure of $f$ in more detail.

Let $\gamma$ designate a simple closed rectifiable Jordan curve and let $H(z)$ be analytic and single valued in an annular region $A$ which contains $\gamma$ in its interior. Let $I$ and $E$ designate the bounded and unbounded regions into which the plane is divided by $\gamma$. The Cauchy integral

$$
t(z)=\frac{1}{2 \pi i} \int_{\gamma} \frac{H(w)}{z-w} d w
$$

defines two analytic functions $t_{E}(z)$ and $t_{I}(z)$ in $E$ and $I$ respectively.

Lemma. $H(z)-t_{E}(z)$ is continuable analytically to $I$ and $H(z)-t_{I}(z)$ is continuable analytically to $E$.

Proof. Let contours $\gamma_{I}$ and $\gamma_{E}$ lie in $A$ and surround $\gamma$ on the $I$ and on the $E$ sides respectively. Let $z$ lie in $I$, but within the annulus bounded by $\gamma_{I}$ and $\gamma$. By Cauchy's theorem, we have 


$$
H(z)=\frac{1}{2 \pi i} \int_{\gamma} \frac{H(w)}{z-w} d w-\int_{\gamma_{I}} \frac{H(w)}{z-w} d w .
$$

Thus,

$$
H(z)-t_{I}(z)=-\int_{\gamma_{I}} \frac{H(w)}{z-w} d w=-t_{E}(z) .
$$

The functions $t_{I}$ and $t_{E}$ are frequently called the inner and outer functions for $H$.

We can now establish that the singularity structure of $f_{j}(z)$ is identical to that of $\bar{h}\left(S_{j}(z)\right)$.

THEOREM 2. The function $f_{j}(z)-\bar{h}\left(S_{j}(z)\right)$ which is initially known to be analytic in an annular strip in $B_{j}$ adjacent to $b_{j}$ may be continued analytically across $b_{j}$ and into the whole interior of $B$.

Proof. From (21) we have

$$
\begin{aligned}
f_{j}(z) & =\frac{1}{2 \pi i} \sum_{k=0}^{N} \int_{b_{k}} \frac{\bar{h}\left(S_{k}(w)\right)}{z-w} d w, \\
& =\frac{1}{2 \pi i}\left\{\int_{b_{j}} \frac{\bar{h}\left(S_{j}(w)\right)}{z-w} d w+\sum_{k \neq j} \int_{b_{k}} \frac{\bar{h}\left(S_{k}(w)\right)}{z-w} d w\right\} .
\end{aligned}
$$

Now, the function $\bar{h}\left(S_{j}(w)\right)$ is regular on $b_{j}$ and hence by our Lemma, $\int_{b_{j}}\left(\bar{h}\left(S_{j}(w)\right) /(z-w)\right) d w-\bar{h}\left(S_{j}(w)\right)$ may be continued analytically across $b_{j}$ to the complete interior of $B$. On the other hand, consider the remaining integrals $\int_{b_{k}}\left(\bar{h}\left(S_{k}(w)\right) /(z-w)\right) d w, z \in B_{j}, k \neq j$. These can obviously be continued analytically from $B_{j}$ into the complete interior of $B$. However, due to the presence of the contour $b_{k}$ itself, this continuation may possibly not be singlevalued. We now prove that the period around each contour is zero and hence the integrals are single-valued. Let $c_{k}$ be a contour lying in $B$ and surrounding $b_{k}$. Then,

$$
\begin{aligned}
& \Delta_{c_{k}} \int_{b_{k}} \frac{\bar{h}\left(S_{k}(w)\right)}{z-w} d w=\int_{c_{k}}\left[\frac{d}{d z} \int_{b_{k}} \frac{\bar{h}\left(S_{k}(w)\right) d w}{z-w}\right] d z \\
& \quad=-\int_{c_{k}} d z \int_{b_{k}} \frac{\bar{h}\left(S_{k}(w)\right)}{(z-w)^{2}} d w=-\int_{b_{k}} \bar{h}\left(S_{k}(w)\right) \int_{c_{k}} \frac{d z}{(z-w)^{2}} d w=0
\end{aligned}
$$

since the inner integral vanishes identically for $w \in b_{k}$. This completes the proof.

We now wish to refer the continuation properties of $f$ to the mapping functions $m_{k}$ instead of to the Schwarz functions $S_{k}$. We first introduce some geometrical notations. 

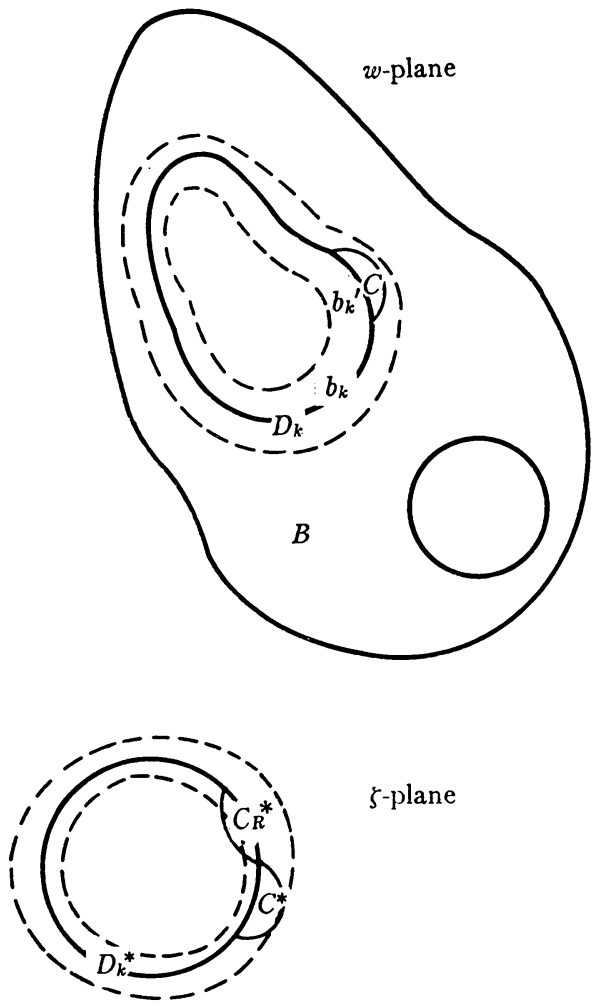

Fig. 2a

As in $\S 2$, each boundary $b_{k}$ is surrounded by an annular strip $D_{k}$ $(k=0,1, \cdots, N)$ in which the mapping functions $\zeta=M_{k}(w), w=m_{k}(\zeta)$ are regular and schlicht. Let $C$ be any region which lies in $B \cap D_{k}$ (for fixed $k$ ) and which possesses part of $b_{k}$ as part of its boundary (see Figs. 2a, 2b). Let this part of the boundary of $b_{k}$ be designated by $b_{k}^{\prime}$. Under the map $M_{k}$, $C$ goes into a region $C^{*}$ which is contained in the image of $D_{k}$ and which borders on $|\zeta|=1$. Let this part of $|\zeta|=1$ be called $c^{\prime}$. Under reflection in $|\zeta|=1$, the region $C^{*}$ goes into a region $C_{R}^{*}$ which borders on $|\zeta|=1$. The region $C_{R}^{*}$ may or may not be contained in the image $D_{k}^{*}$ of $D_{k}$ under $M$.

Having fixed an $M_{k}$, we may set up the correspondence $C \leftrightarrow C_{R}^{*}$ (depending upon $M_{k}$ ) as explained above. In the theorem which follows, the regions $C$ and $C_{R}^{*}$ will have this meaning.

THEOREM 3. The transform $f_{k}(z)$ can be continued analytically across $b_{k}^{\prime}$ so as to be regular in $B_{k} \cup C$ if and only if $\bar{h}\left(\bar{m}_{k}(z)\right)$ can be continued analytically so as to be regular in $C_{R}^{*}$.

Proof. Suppose first that $f_{k}(z)$ can be continụed analytically across $b_{k}^{\prime}$ so 

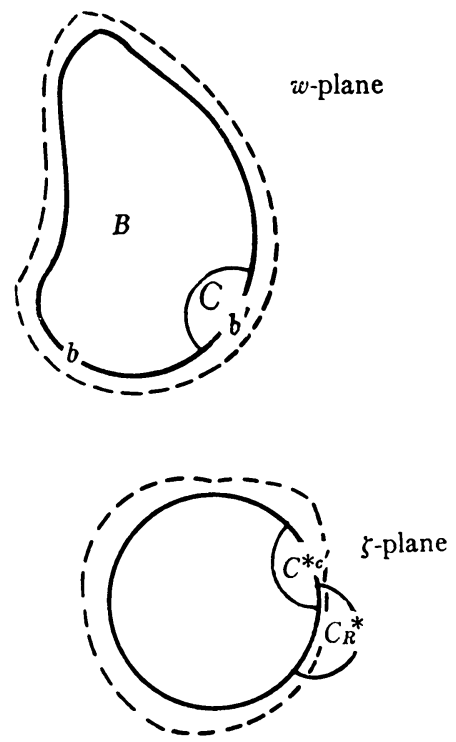

FIG. 2b. Simply connected region utilizing inner mapping function.

as to be regular in $B_{k} \cup C$. By Theorem $2, f_{k}(z)-\bar{h}\left(\bar{m}_{k}\left(1 / M_{k}(z)\right)\right)$ is regular in $B$. Hence, $\bar{h}\left(\bar{m}_{k}\left(1 / M_{k}(z)\right)\right)$ can be continued across $b_{\boldsymbol{k}}^{\prime}$ so as to be regular in $C$. Designate this composite function by $p(z)$. As $z$ varies in $C, 1 / M_{k}(z)$ varies in $C_{R}^{*}$. Set $t=1 / M_{k}(z)$, then for $t \in C_{R}^{*}, z=m_{k}(1 / t)$. Thus, $z$ is a regular function of $t$ for $t \in C_{R}^{*}$. Hence $p\left(m_{k}(1 / t)\right)$ is a regular function of $t$ in $C_{R}^{*}$. But, $p\left(m_{k}(1 / t)\right)$ $=\bar{h}\left(\bar{m}_{k}\left(1 / M_{k}\left(m_{k}(1 / t)\right)\right)\right)=\bar{h}\left(\bar{m}_{k}(t)\right)$. Thus, $\bar{h}\left(\bar{m}_{k}(t)\right)$ is regular in $C_{R}^{*}$.

Conversely, let $\bar{h}\left(\bar{m}_{k}(t)\right)$ continue analytically so as to be regular in $C_{R}^{*}$. For $z \in C, M_{k}(z)$ is regular and takes values in $C^{*}$, while $1 / M_{k}(z)$ takes values in $C_{R}^{*}$. Therefore, $\bar{h}\left(\bar{m}_{k}\left(1 / M_{k}(z)\right)\right)$ is regular in $C$. But by Theorem $2, f_{k}(z)$ $-\bar{h}\left(\bar{m}_{k}\left(1 / M_{k}(z)\right)\right)$ is regular in $B$. Therefore $f_{k}(z)$ must be regular in $C$.

In this theorem as it is phrased above, the region $C$ must be taken interior to $D_{k}$. However, if by $M_{k}$ we understand an interior mapping function of $b_{k}(k=0)$ and an exterior mapping function $(k \geqq 1)$, then any $C$ lying in $B$ and bordering on $b_{k}$ will be admissible.

COROLlary. Let $B$ be a simply connected region and let $m(z)$ map the interior of the unit circle onto $B$ such that $m(0)=0$. Then, $f(z)=f_{0}(z)$ continues analytically into $B-(0)$ if and only if $\bar{h}(\bar{m}(z))$ is an entire function.

Proof. Under the correspondence $C \leftrightarrow C_{R}^{*}$ (see Fig. 2b), any simply connected region lying in $B$, bordering on an arc of $b$ and not containing the origin corresponds to a finite simply connected region lying in $|\zeta|>1$, bordering on an arc of $|\zeta|=1$, and vice-versa. The corollary now follows.

Additional corollaries can be obtained by specializing the function $g$. 
Thus, e.g. if $g(z) \equiv 1$, then the above results read directly on $m(z)$.

8. Analytic continuation and moment properties. In the present section, we relate the analytic continuability of the mapping function of a simply connected domain with the growth properties of certain sequences of moments.

TheOREM 4. Let $B$ be simply connected and let $m(z)$ map the unit circle onto $B$ with $m(0)=0 . A$ necessary and sufficient condition that $\bar{h}(\bar{m}(z))$ be an entire function is that

$$
\lim _{n \rightarrow \infty}\left|\iint_{B} z^{n} g(z) d A\right|^{1 / n}=0
$$

Proof. From the previous Corollary, a necessary and sufficient condition that $\bar{h}(\bar{m}(z))$ be entire is that $f(z)$ continue analytically to $z=0$. From (25) and the Cauchy-Hadamard formula for the radius of convergence of a power series, we obtain (55).

Corollary. Let $m(z)$ be defined as above. A necessary and sufficient condition that $[m(z)]^{k+1}$ be an entire function is that

$$
\lim _{n \rightarrow \infty}\left|\iint_{B} z^{n}\left[\bar{z}^{k}\right]-d A\right|^{1 / n}=0 .
$$

A necessary and sufficient condition that $m(z)$ be entire is that

$$
\lim _{n \rightarrow \infty}\left|\iint_{B} z^{n} d A\right|^{1 / n}=0 .
$$

In the case of multiply connected domains, Theorem 4 is no longer applicable, but the following inequality may be obtained. See also (86), (87).

THEOREM 5. Let $m_{k}(\zeta)$ map the interior of $|\zeta|=1$ conformally onto the interior of $b_{k}(k=0,1, \cdots, N)$ with $m_{k}(0)=a_{k}$. If $\bar{h}\left(\bar{m}_{k}(\zeta)\right)$ are all entire functions, then

$$
\limsup _{n \rightarrow \infty}\left|\iint_{B} z^{n}[g(z)]^{-} d A\right|^{1 / n} \leqq \max _{0 \leqq k \leqq N}\left|a_{k}\right|
$$

Proof. From $\left(27^{\prime}\right)$ we have,

$$
\frac{1}{\pi} \iint_{B} z^{n}[g(z)]-d A=\frac{1}{2 \pi i} \int_{|\zeta|=1} \sum_{k=0}^{N}\left\lfloor m_{k}(\zeta)\right]^{n} \bar{h}\left(\bar{m}_{k}\left(\frac{1}{\zeta}\right)\right) m_{k}^{\prime}(\zeta) d \zeta .
$$

In view of the fact that the functions $\bar{h}\left(\bar{m}_{k}(\zeta)\right)$ are entire, the integration path in the last integral may be taken as $|\zeta|=r$, for all $0<r \leqq 1$. Let $A_{k}(r)$ $=\max _{|\zeta|=r}\left|m_{k}(\zeta)\right|, B_{k}(r)=\max _{|\zeta|=r}\left|\bar{h}\left(\bar{m}_{k}(1 / \zeta)\right) m_{k}^{\prime}(\zeta)\right|$. Then, 


$$
\frac{1}{\pi}\left|\iint_{B} z^{n}[g(z)]-d A\right| \leqq r \sum_{k=0}^{N}\left[A_{k}(r)\right]^{n} B_{k}(r),
$$

and

$$
\left|\iint_{B} z^{n}[g(z)]-d A\right|^{1 / n} \leqq(\pi r)^{1 / n}\left\{\sum_{k=0}^{N}\left[A_{k}(r)\right]^{n} B_{k}(r)\right\}^{1 / n} .
$$

Therefore,

$$
\underset{n \rightarrow \infty}{\limsup }\left|\iint_{B} z^{n}[g(z)]-d A\right|^{1 / n} \leqq \max _{0 \leqq k \leqq N} A_{k}(r) .
$$

Since $r$ may be selected arbitrarily small and since $\lim _{r \rightarrow 0} A_{k}(r)=\left|a_{k}\right|$, we now obtain (57).

Corollary. If $B$ is doubly connected and if $m_{k}(0)=0(k=0,1)$ and $\bar{h}\left(\bar{m}_{k}(\zeta)\right)$ are entire then

$$
\lim _{n \rightarrow \infty}\left|\iint_{B} z^{n}[g(z)]-d A\right|^{1 / n}=0 .
$$

In the two theorems which follow, we deal with simply connected regions $B$ with interior mapping function $m(\zeta), m(0)=0$.

THEOREM 6. The function $h(m(\zeta))$ is a polynomial of degree $n$ if and only if

$$
\iint_{B} z^{p}[g(z)]-d A=0, \quad p \geqq n .
$$

Proof. If $t(\zeta)=\bar{h}(\bar{m}(\zeta))$, then $[t(\bar{\zeta})]^{-}=h(m(\zeta))$; hence, $h(m(\zeta))$ is a polynomial of degree $n$ if and only if $t(\zeta)$ is. Write $m(\zeta)=a_{1} \zeta+a_{2} \zeta^{2}+\cdots, m^{\prime}(\zeta)$ $=a_{1}+2 a_{2} \zeta+\cdots$ and assume first of all that $\bar{h}(\bar{m}(\zeta))=b_{0}+b_{1} \zeta+\cdots+b_{n} \zeta^{n}$.

From $\left(27^{\prime}\right)$ we have

$$
\frac{1}{\pi} \iint_{B} z^{p}[g(z)]^{-} d \Lambda=\frac{1}{2 \pi i} \int_{|\zeta|=1}[m(\zeta)]^{p} \bar{h}\left(\bar{m}\left(\frac{1}{\zeta}\right)\right) m^{\prime}(\zeta) d \zeta .
$$

Now $[m(\zeta)]^{p} m^{\prime}(\zeta)=\zeta^{p} R_{p}(\zeta)$ where $R_{p}$, is some regular function in $|\zeta|=1$, and $R_{p}(0) \neq 0$. Then,

$$
\frac{1}{\pi} \iint_{B} z^{p}[g(z)]-d A=\frac{1}{2 \pi i} \int_{|\zeta|=1} \zeta^{p} R_{p}(\zeta)\left[b_{0}+\frac{b_{1}}{\zeta}+\cdots+\frac{b_{n}}{\zeta^{n}}\right] d \zeta
$$

For $p \geqq n$ the right hand integrand is regular in $|\zeta|=1$ and (62) follows.

Conversely, suppose that (62) holds. Then by (25) we have

$$
f(z)=\frac{c_{0}}{z}+\frac{c_{1}}{z^{2}}+\cdots+\frac{c_{n}}{z^{n}}
$$


for some constants $c_{0}, c_{1}, \cdots, c_{n}$. On the other hand, from (37) we have

$$
f(z)=\frac{1}{\pi} \sum_{n=0}^{\infty}\left(\iint_{B}[g(w)]-\Phi_{n}^{*}(w) d A\right) \frac{1}{z} P_{n}\left(\frac{1}{z}\right) .
$$

In view of the fact that $P_{n}(z)$ are polynomials of degree $n$, we have from (64) and for some constants $d_{0}, d_{1}, \cdots, d_{n-1}$,

$$
f(z)=d_{0} \frac{1}{z} P_{0}\left(\frac{1}{z}\right)+d_{1} \frac{1}{z} P_{1}\left(\frac{1}{z}\right)+\cdots+d_{n-1} \frac{1}{z} P_{n-1}\left(\frac{1}{z}\right) .
$$

It follows from (65), (66), (36) and the uniqueness of the representation of $f$ in a series of $P_{n}$ 's that

$$
\iint_{B}[g(w)]^{-} \Phi_{p}^{*}(w) d A=0 \quad \text { for } p \geqq n
$$

Using $\left(29^{\prime}\right)$ we have

$$
\iint_{B}[g(w)]^{-} M^{\prime}(w)[M(w)]^{p} d A=0 \quad \text { for } p \geqq n .
$$

Applying Green's Theorem to (68) there is obtained

$$
\int_{b}[h(w)]^{-} M^{\prime}(w)[M(w)]^{p} d w=0 \quad \text { for } p \geqq n
$$

or

$$
\int_{|\zeta|=1}[h(m(\zeta))]^{-} \zeta^{p} d \zeta=0 \quad \text { for } p \geqq n .
$$

Since by Theorem $4 h(m(\zeta))$ is entire, it is regular in $|\zeta| \leqq 1$ and it follows from $(70)$ that $h(m(\zeta))$ must be a polynomial of degree $n$ at most.

Corollaries. The mapping function $m(\zeta), m(0)=0$, has the form $m(\zeta)$ $=(\text { Polyn }(\zeta))^{1 / k}$ if and only if $\iint_{B} z^{n} \bar{z}^{k-1} d A=0$ for all $n$ sufficiently large. $m(\zeta)$ is a polynomial if and only if $\iint_{B} z^{n} d A=0$ for all $n$ sufficiently large. $B$ is the circle $|z|=r$ if and only if $\iint_{B} z^{n} d A=0, n=1,2,3, \cdots$.

Theorem 7. Define $r(z)=(1 / z) f(1 / z)$. The function $r(z)$ is entire of order $\leqq \alpha$ if and only if $h(m(\zeta))$ is entire of order $\leqq \alpha$. Here $m(0)=0$.

Proof. If $t(\zeta)=\bar{h}(\bar{m}(\zeta))$, then $h(m(\zeta))=[t(\bar{\zeta})]$-. Hence, $h(m(\zeta))$ is entire of order $\leqq \alpha$ if and only if $t(\zeta)$ has this same property.

From (12) we have,

$$
r(z)=\frac{1}{2 \pi i} \int_{|\zeta|=1} \frac{\bar{h}(\bar{m}(1 / \zeta)) m^{\prime}(\zeta) d \zeta}{1-z m(\zeta)} .
$$


To establish an inversion formula for (71), we could use Theorem 1. However, it seems simpler to proceed as follows. From (40) we have

$$
\int_{b} \Phi_{n}^{*}(z) f(z) d z=2 i \iint_{B}[g(z)]^{-} \Phi_{n}^{*}(z) d A .
$$

Utilizing $\left(29^{\prime}\right)$

$$
\begin{aligned}
\int_{b} M^{\prime}(z)[M(z)]^{n} f(z) d z & =2 i \iint_{B}[g(z)]^{-} M^{\prime}(z)[M(z)]^{n} d A \\
& =\int_{b}[h(z)]^{-} M^{\prime}(z)[M(z)]^{n} d z,
\end{aligned}
$$

the last equality following by application of Green's theorem. Here $M$ is the interior mapping function of $B$ and is inverse to $m$.

Applying the transformation $z=m(\zeta)$ to the first and last integrals in (73) we obtain

$$
\int_{|\zeta|=1} \zeta^{n} \frac{1}{m(\zeta)} r\left(\frac{1}{m(\zeta)}\right) d \zeta=\int_{|\zeta|=1}[h(m(\zeta))]^{-} \zeta^{n} d \zeta
$$

Now if $h(m(\zeta))=\sum_{k=0}^{\infty} d_{k} \zeta^{k}$, then

$$
\int_{|\zeta|=1}[h(m(\zeta))]^{-} \zeta^{n} d \zeta=2 \pi i \bar{d}_{n+1}
$$

so that

$$
\begin{aligned}
{[h(m(w))]^{-}=\sum_{k=0}^{\infty} \bar{d}_{k} \bar{w}^{k} } & =\frac{1}{2 \pi i} \sum_{k=0}^{\infty} \bar{w}^{k} \int_{|\zeta|=1}[h(m(\zeta))]-\zeta^{k-1} d \zeta \\
& =\frac{1}{2 \pi i} \sum_{k=0}^{\infty} \bar{w}^{k} \int_{|\zeta|=1} \frac{\zeta^{k}}{\zeta} \frac{1}{m(\zeta)} r\left(\frac{1}{m(\zeta)}\right) d \zeta \\
& =\frac{1}{2 \pi i} \int_{|\zeta|=1} \frac{r(1 / m(\zeta)) d \zeta}{\zeta(1-\bar{w} \zeta) m(\zeta)}
\end{aligned}
$$

which is the required inversion formula.

Suppose now that $\bar{h}(\bar{m}(\zeta))$ is entire and satisfies an inequality of the form

$$
|\bar{h}(\bar{m}(\zeta))|<\phi(r), \quad 0<r=|\zeta|<\infty .
$$

Since $\bar{h}(\bar{m}(\zeta))$ is entire, then the integral in (71) may be extended over any circle whose radius is sufficiently small. For each $z$, we shall select an appropriate radius $r_{z}$ and obtain from (71) and (76) the estimate

$$
|r(z)| \leqq r_{z} \cdot \phi\left(\frac{1}{r_{z}}\right) \cdot A \cdot \max _{|\zeta|=r_{z}}\left|\frac{1}{1-z m(\zeta)}\right|
$$


where $A=\max _{|\zeta| \leq 1}\left|m^{\prime}(\zeta)\right|$.

Since $m(\zeta)=a_{1} \zeta+a_{2} \zeta^{2}+\cdots, \lim _{\zeta \rightarrow 0} m(\zeta) / \zeta=a_{1}$. Hence to a given $\epsilon$, there is a $\zeta_{0}$ such that

$$
\left(\left|a_{1}\right|-\epsilon\right)|\zeta| \leqq|m(\zeta)| \leqq\left(\left|a_{1}\right|+\epsilon\right)|\zeta|
$$

for $|\zeta| \leqq \zeta_{0}$. Fix a $0<\epsilon<\left|a_{1}\right|$ and select $r_{z}$ as follows,

$$
r_{z}=\frac{1}{(1+\epsilon)\left(\left|a_{1}\right|-\epsilon\right)|z|} .
$$

For $z$ sufficiently large, $r_{z}<\zeta_{0}$, and hence, from (78) and (79),

$$
\min _{|\zeta|=r_{z}}|m(\zeta)| \geqq \frac{1}{(1+\epsilon)|z|} .
$$

Hence, $\max _{|\zeta|=r_{z}}|1 /(1-z m(\zeta))| \leqq(1+\epsilon) / \epsilon$. Thus, for $|z|$ sufficiently large,

$$
|r(z)| \leqq \frac{A}{\epsilon\left(\left|a_{1}\right|-\epsilon\right)|z|} \phi\left((1+\epsilon)\left(\left|a_{1}\right|-\epsilon\right)|z|\right) .
$$

Assuming, conversely, that $r(z)$ is entire and satisfies an inequality of the type

$$
|r(z)| \leqq \psi(r), \quad 0<r=|z|<\infty,
$$

we may proceed similarly with the integral (75). Select

$$
r_{w}=\frac{1}{(1+\epsilon)|w|} .
$$

For $|w|$ sufficiently large, $r_{w}<\zeta_{0}$ and therefore

$$
\min _{|\zeta|=r_{w}}|m(\zeta)| \geqq \frac{\left|a_{1}\right|-\epsilon}{(1+\epsilon)|w|},
$$

while $\max _{|\zeta|=r_{w}}|1 /(1-\bar{w} \zeta)| \leqq(1+\epsilon) / \epsilon$. Then, from (75), for $|w|$ sufficiently large,

$$
|h(m(w))| \leqq \frac{(1+\epsilon)^{2}|w|}{(\epsilon)\left(\left|a_{1}\right|-\epsilon\right)} \psi\left(\frac{(1+\epsilon)|w|}{\left|a_{1}\right|-\epsilon}\right) .
$$

If we now select, as a special choice, $\phi=\psi=e^{\sigma r^{\alpha}}$, the theorem follows from (80) and (83).

CoROLlaRy. The function $h(m(\zeta))$ is entire of order $<a$ if and only if

$$
\left|\iint_{B} z^{n}[g(z)]-d A\right|^{1 / n}=O\left(n^{-1 / \alpha}\right), \quad n \rightarrow \infty .
$$


Proof. The quantities $\iint_{B} z^{n}[g(z)]-d A$ are the Taylor coefficients of $r(z)$ so that classical growth theorems $\left({ }^{6}\right)$ may be employed.

9. Identification of $\lim _{n \rightarrow \infty} \sup \left|\iint_{B} z^{n} g(z) d A\right|^{1 / n}$. We have seen in the previous section that growth properties of the quantities $\left|\iint_{B} w^{n}[g(w)]-d A\right|^{1 / n}$ are intimately related to the analytic continuability of $h(m(\zeta))$. Introduce the abbreviation

$$
\sigma=\sigma_{g}=\limsup _{n \rightarrow \infty}\left|\iint_{B} z^{n}[g(z)]-d A\right|^{1 / n} .
$$

In $\$ 8$ we investigated the case $\sigma=0$. In the present section we shall study the case $\sigma>0$ in more detail.

If $R$ designates the maximum distance from 0 to $\hat{B}$, then (85) yields the immediate estimate

$$
\sigma \leqq R .
$$

In many cases the weak inequality in (86) can be strengthened. Let $m_{0}(\zeta)$ map the interior of $|\zeta|=1$ conformally onto the interior of $C_{0}$, and assume that $\bar{h}\left(\bar{m}_{0}(\zeta)\right)$ is regular in $|\zeta| \leqq r, r>1$. Then, using the same technique as that employed in the proof of Theorem 5 , we can show that

$$
\sigma \leqq \max _{|\zeta|=r^{-1}}\left|m_{0}(\zeta)\right|
$$

Hence, in this case

$$
\sigma<R
$$

Thus, the equality sign in (86) can hold only if $\bar{h}\left(\bar{m}_{0}(z)\right)$ is nonanalytic on the outer boundary. This occurs, e.g., in the case of the unit square $S$ with $g \equiv 1$. In this case, an explicit computation yields $\sigma=2^{1 / 2}=R$. The converse is not true. There are simply connected domains with a nonanalytic boundary for which $\sigma<R$. Thus, for instance, let $B$ be the crescent shaped region formed by the two circles $c_{1}:|z| \leqq 1, c_{2}:|z-1 / 2| \leqq 1 / 2$. We have, for $n>0$,

$$
\iint_{B} z^{n} d A=\iint_{c_{1}} z^{n} d A-\iint_{c_{2}} z^{n} d A=-\iint_{c_{2}} z^{n} d A .
$$

Now

$$
\lim \left|\iint_{B} z^{n} d A\right|^{1 / n}=\lim \left|\iint_{c_{2}} z^{n} d A\right|^{1 / n}=\frac{1}{2}<1=R .
$$

It is of considerable interest to identify the constant $\sigma_{g}$ more precisely for simply connected domains with an analytic boundary. To this end, we make the following definitions. We assume here that $B$ contains the origin.

(6) Cf., for example, Titchmarsh, [6, p. 253]. 

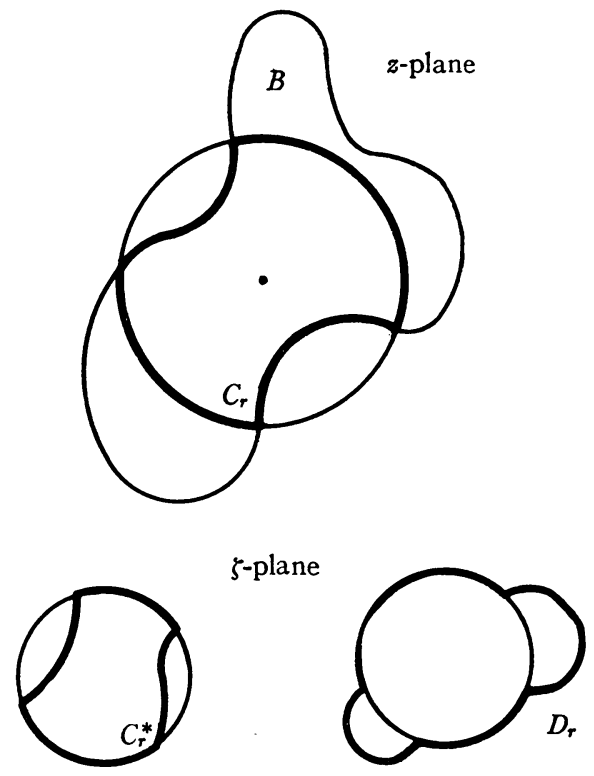

FIG. 3

For values of $r, 0 \leqq r \leqq R=\max _{z} \in \widehat{B}|z|$, define a one-parameter family of curves $C_{r}$ as follows, (see Fig. 3).

$$
C_{r}=\text { boundary of }[B \cap(|z| \leqq r)] \text {. }
$$

The curve $C_{r}$ is therefore contained in $\hat{B}$ and, under the interior map $M(z)$ has an image $C_{r}^{*}$. Under the $\operatorname{map} \zeta^{\prime}=1 / \zeta, C_{r}^{*}$ has an image $D_{r}$. For small values of $r, D_{r}$ is a very large circle-like curve which tends to $\infty$ as $r \rightarrow 0$. As $r \rightarrow R, D_{r}$ becomes the circle $|\zeta|=1$ itself. If $r_{1}<r_{2}$, it is clear that $D_{r_{1}}$ contains $D_{r_{2}}$ in its interior. It should be emphasized that the family $D_{r}$ is a fixed set of curves which depend upon $B$, but not upon $g$. If $g$ is analytic in $\hat{B}$, then $\bar{h}(\bar{m}(\zeta))$ is analytic in $|\zeta| \leqq 1$ and therefore, for values of $r$ sufficiently close to $R$, it can be continued analytically to $D_{r}$. As $r \rightarrow 0$, and $D_{r}$ expands, this property will, in general, cease. We make the following definition: $r_{0}$ is the greatest lower bound of quantities $r$ such that $\bar{h}(\bar{m}(z))$ is continuable analytically to $D_{r}$.

THEOREM 8. $\sigma_{g}=r_{0}$.

Proof. From (25) we have $f(z)=(1 / \pi) \sum_{n=0}^{\infty} z^{-n-1} \iint_{B} w^{n}[g(w)]-d A$. From (85) and the Cauchy-Hadamard theorem, $f(z)$ must be regular in the region $|z|>\sigma_{g}$ and will possess a singularity on $|z|=\sigma_{g}$. Thus, $f(z)$ will continue across certain parts of the boundary of $B$ and will be regular in $C$ $=B-\left(|z| \leqq \sigma_{g}\right)$. By Theorem 3 it follows that $\bar{h}(\bar{m}(\zeta))$ must be regular in the region $C_{R}^{*}$ corresponding to $C$. (The correspondence of Theorem 3.) This $C_{R}^{*}$ is 
precisely $D_{\sigma_{g}}$ as defined in the previous paragraph. Hence $r_{0} \leqq \sigma_{g}$. Suppose now $r_{0}<\sigma_{g}$. Then, by definition, $\bar{h}(\bar{m}(\zeta))$ would be continuable analytically to $D_{r_{0}}$ and hence again by Theorem $3, f(z)$ would be regular in the region $B-\left(|z| \leqq r_{0}\right)$, which is a larger region than $C$. Now from (4) it is clear that $f(z)$ can have no singularity in the complement of $\hat{B}$. Hence, the singularity of $f$ which is located on $|z|=\sigma_{o}$ must occur either at a boundary or an interior point of $B$. From Theorem 1 , since $g$ is analytic on $b, f$ must be regular on $b$. Therefore the singularity on $|z|=\sigma_{0}$ is located at an interior point. This contradicts the regularity of $f$ in $B-\left(|z| \leqq r_{0}\right), r_{0}<\sigma_{o}$. Therefore we must have $r_{0}=\sigma_{g}$.

10. The Schwarz reflection function; further facts. The function $S(z)$ defined by

$$
S(z)=\bar{z} ; \quad z \text { on } b
$$

is sufficiently interesting in its own right to warrant separate study. This function embodies within it the reflection principle for analytic functions in that the following facts are true.

(a) The point $z_{R}$ given by

$$
z_{R}=[S(z)]^{-}
$$

is the reflection in $b$ of the point $z$. Given a small neighborhood containing an arc of $b$, let $w=t(z)$ map this neighborhood one to one conformally in such a way that $b$ goes into a part of the real axis $\operatorname{Im}(w)=0$. According to Schwarz, the reflection of $z$ in $b$ is given by $z_{R}=t^{-1}[(t(z))]$-. Consider the function $S_{1}(z)=\left[t^{-1}[(t(z))]^{-}\right]^{-}$. For $z$ on $b$, we have $S_{1}(z)=\bar{z}$. Therefore $S_{1}(z)=S(z)$ and (91)holds.

(b) If $f(z)$ is regular in $B$ and continuous in $\hat{B}$ and if for $z$ on $b, f(z)$ takes values on the unit circle, then $f(z)$ may be continued analytically across $b$ by means of the formula

$$
f[(S(z))]^{-}=1 /[f(z)]^{-} .
$$

(c) More generally, the invariant form of (92) is as follows. Let $f(z)$ be regular in $B$ and continuous in $\hat{B}$ and for $z \in b$, let $f(z)$ take on values on an analytic curve $d$. Designate the Schwarz functions of $b$ and $d$ by $S_{b}$ and $S_{d}$ respectively. Then $f(z)$ may be continued analytically across $b$ by means of the formula

$$
f\left(\left[S_{b}(z)\right]^{-}\right)=\left[S_{d}(f(z))\right]^{-} .
$$

This may be written in the form,

$$
S_{d}(z)=\bar{f}\left(S_{b}\left(f^{-1}(z)\right)\right)
$$

which is the law of transformation of one reflection function into another.

(d) $S(z)$ satisfies the equation 


$$
\bar{S}(S(z))=z,
$$

identically. The "Hermitian idempotence" of $S(z)$ expresses the idempotence of the reflection operation.

The differential geometry of reflection has been studied by E. Kasner $\left({ }^{7}\right)$. The identity (95) is reminiscent of the theory of permutable functions as developed by $\operatorname{Ritt}\left({ }^{8}\right)$ and others.

We have already seen that an analytic curve determines $S(z)$ uniquely. The converse is also true:

TheOREM. Let $S(z)$ be regular and single valued in a doubly connected annulus-like region $A$. Then $S(z)$ cannot be the Schwarz function for two distinct homotopic analytic curves lying in $A$.

Proof. Let $C_{1}$ and $C_{2}$ be two analytic curves lying in $A$ and bounding the (finite) simply connected regions $B_{1}$ and $B_{2}$ respectively. Assume that $S(z)$ is the Schwarz function for both $C_{1}$ and $C_{2}$. As a special case of (27), or by applying the complex Green's theorem directly to the left hand number, we have

$$
\begin{array}{r}
\iint_{B_{1}} z^{m \bar{z}^{n}} d A=\frac{1}{2 i(n+1)} \int_{C_{1}} z^{m \bar{z}^{n+1}} d z=\frac{1}{2 i(n+1)} \int_{C_{1}} z^{m}[S(z)]^{n+1} d z \\
(m, n=0,1, \cdots) .
\end{array}
$$

Similarly

$$
\iint_{B_{2}} z^{m} \bar{z}^{n} d A=\frac{1}{2 i(n+1)} \int_{C_{2}} z^{m}[S(z)]^{n+1} d z .
$$

By the assumed regularity of $S(z)$ in $A$, we have by Cauchy's theorem,

$$
\int_{C_{1}} z^{m} S^{n+1}(z) d z=\int_{C_{2}} z^{m} S^{n+1}(z) d z
$$

Therefore,

$$
\iint_{B_{1}} z^{m} \bar{z}^{n} d A=\iint_{B_{2}} z^{m \bar{z}^{n}} d A, \quad(m, n=0,1, \cdots) .
$$

Therefore, from (3b),

$$
\iint_{B_{1}} x^{m} y^{n} d A=\iint_{B_{2}} x^{m} y^{n} d A \quad(m, n=0,1, \cdots) .
$$

From the corollary in the introduction it follows that $B_{1}=B_{2}$.

(7) Annals of Math. vol. 38 (1937).

(8) Comptes Rendus vol. 176 (1923). 
Though the singularity structure of $S(z)$ is completely given by Theorem 2 , it is of interest to obtain results on this question which are independent of the transform $f(z)$. We note first of all that $S(z)$ cannot be regular throughout the interior of its curve. For, from, (95) with $m=n=0$,

$$
0<\operatorname{Area}(B)=\frac{1}{2 i} \int_{C} S(z) d z=0
$$

assuming regularity. We may even state that $S(z)$ cannot be equal to the derivative of a function which is analytic and single valued on $C$. This statement may be generalized in two ways;

TheOREM. Let $g$ be regular in $B$, and let $\bar{g}(S(z))$, initially known to be regular near $C$, continue analytically to be regular in all of $B$. Then $g$ is identically constant.

Proof. We have

$$
\begin{aligned}
\iint_{B}\left|g^{\prime}(z)\right|^{2} d A & =\iint_{B} g^{\prime}(z)\left[g^{\prime}(z)\right]-d A=\frac{1}{2 i} \int_{C} g^{\prime}(z)[g(z)]^{-} d z \\
& =\frac{1}{2 i} \int_{C} g^{\prime}(z) \bar{g}(S(z)) d z=0 .
\end{aligned}
$$

Therefore $g^{\prime}(z)=0$ and the conclusion follows. The same conclusion holds if we require that $g^{\prime}(z) \bar{g}(S(z))$ be the derivative of a function analytic and single valued on $C$.

It follows from this theorem that the singularities of $S(z)$ cannot be annihilated by its being operated upon by a regular function.

Theorem. Let $B$ be contained in $|z|<d$. Let $f(z)$ be analytic in $|z|<d^{2}$ and satisfy $x f^{\prime}(x)+f(x)>0$ for real $x>0$. Then $S(z) f(z S(z))$ cannot be regular in $B$ (or cannot be the derivative of a function which is analytic and single valued on C).

Proof. Let

$$
f(x)=\sum_{n=0}^{\infty} \frac{a_{n}}{n+1} x^{n} \quad|x|<d^{2} .
$$

Then,

$$
r^{2} f\left(r^{2}\right)=\sum_{n=0}^{\infty} \frac{a_{n}}{n+1} r^{2 n} \quad|r|<d
$$

and

$$
\frac{d}{d r}\left[r^{2} f\left(r^{2}\right)\right]=2 r \sum_{n=0}^{\infty} a_{n} r^{2 n}>0
$$


by hypothesis. Setting

$$
\begin{aligned}
r^{2} & =x^{2}+y^{2}, 0<\iint_{B} \sum_{0}^{\infty} a_{n} r^{2 n} d x d y=\iint_{B} \sum_{n=0}^{\infty} a_{n} z^{n} \bar{z}^{n} d x d y \\
& =\sum_{n=0}^{\infty} a_{n} \frac{1}{2 i} \int_{b} z^{n} S^{n+1}(z) d z=\frac{1}{2 i} \int_{b} S(z) f(z S(z)) d z .
\end{aligned}
$$

The conclusion now follows as before. As a corollary we cite: Let $p(z), r(z)$ be regular in $B$ and assume that $p(z)=[r(z)]-$ along $C$. Then $p$ and $r$ are constants. For, $p(z)=[r(z)]^{-}$on $C$. Therefore $p(z)=\bar{r}(\bar{z})$ on $C$ or $p(z)=r(S(z))$. The conclusion now follows the previous theorem.

TheOREM $\left({ }^{9}\right)$. The Schwarz reflection function $S(z)$ of an analytic curve $C$ is rational if and only if $C$ is a circle.

Proof. The circle $\left|z-z_{0}\right|^{2}=\rho^{2}$ possesses the reflection function $S(z)$ $=\left(\rho^{2} /\left(z-z_{0}\right)\right)+\bar{z}_{0}$. Conversely, suppose that $S(z)=R(z)=P(z) / Q(z)$ where $P(z)=a_{0} z^{m}+\cdots, Q(z)=b_{0} z^{n}+\cdots, a_{0} b_{0} \neq 0,(P, Q)=1$. Since we have identically $\bar{R}(R(z))=z$,

$$
\frac{a_{0} R^{m}+\bar{a}_{1} R^{m-1}+\cdots}{\bar{b}_{0} R^{n}+\bar{b}_{1} R^{n-1}+\cdots}=z
$$

or,

$$
\left(\bar{a}_{0} R^{m}+\bar{a}_{1} R^{m-1}+\cdots\right)=z\left(\bar{b}_{0} R^{n}+\bar{b}_{1} R^{n-1}+\cdots\right) .
$$

Case 1. Suppose $m>n$. Then,

$$
\bar{a}_{0} P^{m}+\bar{a}_{1} Q P^{m-1}+\cdots=Q^{m}\left(\bar{b}_{0} R^{n}+\bar{b}_{1} R^{n-1}+\cdots\right) .
$$

Thus, $Q \mid \bar{a}_{0} P^{m}$, and since $(P, Q)=1, Q=$ const. Thus, $R=\alpha_{0} z^{m}+\alpha_{1} z^{m-1}+\cdots$. Since $\bar{R}(R(z))=z$, identically, it follows that

$$
z=\bar{\alpha}_{0}\left(\alpha_{0} z^{m}+\alpha_{1} z^{m-1}+\cdots\right)^{m}+\bar{\alpha}_{1}\left(\alpha_{0} z^{m}+\cdots\right)^{m-1}+\cdots .
$$

Comparing leading coefficients, we find $\alpha_{0} \bar{\alpha}_{0} z^{m^{2}}=z$ and so $m=1, \alpha_{0} \bar{\alpha}_{0}=1$. Then,

$$
R(z)=\alpha z+\beta, \quad \alpha \bar{\alpha}=1, \quad \operatorname{Re}(\bar{\alpha} \beta)=0 .
$$

Case 2. Suppose $m<n$. Then as before, $Q \mid \bar{b}_{0} z P^{n}$, and since $(P, Q)=1$, $Q=$ const. or $Q=$ const. $z$. Since $P$ is of smaller degree than $Q$, we must actually have $R(z)=c / z$. Now $\bar{R}(R(z))=z$ implies $c=\bar{c}$. Thus, in this case,

$$
R(z)=c / z, c \text { real. }
$$

(9) The present theorem utilizes the Hermitian idempotence of the reflection function. Though Hermitian idempotence as such does not appear to have been studied, an equivalent theorem for ordinary idempotence is well known. The authors wish to thank Dr. Morris New man for a fruitful discussion in this area. The present arrangement of this proof is due to him. 
Case 3. Suppose $m=n$. Then

$$
\bar{a}_{0} P^{m}+\bar{a}_{1} Q P^{m-1}+\cdots=z\left(\bar{b}_{0} P^{m}+\bar{b}_{1} Q P^{m-1}+\cdots\right) .
$$

This implies that $\bar{a}_{0}-\bar{b}_{0} z \doteq 0(\bmod Q)$. Thus, $Q=C z+d$ and so $P=a z+b$ for some $a, b, c, d$. Writing

$$
R(z)=\left(\begin{array}{ll}
a & b \\
c & d
\end{array}\right) z
$$

the identity $\bar{R}(R(z))=z$ implies

$$
\left(\begin{array}{ll}
\bar{a} & \bar{b} \\
\bar{c} & \bar{d}
\end{array}\right)\left(\begin{array}{ll}
a & b \\
c & d
\end{array}\right) z=z .
$$

Thus, we need only to look for matrices

$$
M=\left(\begin{array}{ll}
a & b \\
c & d
\end{array}\right)
$$

such that $\bar{M} M=I$. Assume, as we may, that $\operatorname{det} M=1$. Since $\bar{M}=M^{-1}$, this implies that

$$
\left(\begin{array}{ll}
\bar{a} & \bar{b} \\
\bar{c} & \bar{d}
\end{array}\right)=\left(\begin{array}{rr}
d & -b \\
-c & a
\end{array}\right)
$$

so that $\bar{a}=d, \bar{b}=-b, \bar{c}=-c, \bar{d}=a$. Writing $a=a_{1}+i a_{2}, b=b_{1}+i b_{2}, c=c_{1}+i c_{2}$, $d=d_{1}+i d_{2}$, we obtain $a=a_{1}+i a_{2}, b=i b_{2}, c=i c_{2}, d=a-i a_{2}$. Thus,

$$
M=\left(\begin{array}{cc}
a & i b \\
i c & \bar{a}
\end{array}\right),
$$

$a \bar{a}+b c=1 . R(z)$ is now seen to take the form

$$
R(z)=\frac{\rho^{2}}{z-z_{0}}+\bar{z}_{0}
$$

with $\rho=c^{-1}$ and $z_{0}=i \bar{a} / c$.

All the solutions of the functional equation $\bar{R}(R(z))=z$ are given by (104), (105), and (106). Case 1 yields the straight lines in the plane, while cases 2 and 3 are the proper circles.

\section{BiBLIOGRAPHY}

1. S. Bergman and M. Schiffer, Kernel functions and conformal mapping, Compositio Math. vol. 8 (1951) pp. 205-249.

2. I. E. Block, Kernel functions and class $L^{2}$, Proc. Amer. Math. Soc. vol. 4 (1953) pp. 110-117.

3. S. Bergman, The kernel function and conformal mapping, Math. Survey's, no. 5, New York, 1950. 
4. P. Davis and H. Pollak, Complex biorthogonality for certain sets of polynomials, Duke Math. J. vol. 21 (1954) pp. 653-668.

5. P. Davis and J. L. Walsh, On representations and extensions of bounded linear functionals defined on classes of analytic functions, Trans. Amer. Math. Soc. vol. 76 (1954) pp. 190-206.

6. E. C. Titchmarsh, The theory of functions, 2d ed., Oxford, 1939.

7. J. L. Walsh and P. Davis, Interpolation and orthonormal systems, Journal d'Analyse Mathematique (1952) pp. 1-28.

The John Simon Guggenheim Foundation,

NEW YoRK, N. Y.

The Bell Telephone Laboratories,

Murray Hill, N. J. 